\title{
ORIGINAL ARTICLE Protein tyrosine kinase 6 regulates mammary gland tumorigenesis in mouse models
}

\author{
M Peng, SM Ball-Kell, RR Franks, H Xie and AL Tyner
}

\begin{abstract}
Protein tyrosine kinase 6 (PTK6, also called BRK) is an intracellular tyrosine kinase expressed in the majority of human breast tumors and breast cancer cell lines, but its expression has not been reported in normal mammary gland. To study functions of PTK6 in vivo, we generated and characterized several transgenic mouse lines with expression of human PTK6 under control of the mouse mammary tumor virus (MMTV) long terminal repeat. Ectopic active PTK6 was detected in luminal epithelial cells of mature transgenic mammary glands. Lines expressing the MMTV-PTK6 transgene exhibited more than a two-fold increase in mammary gland tumor formation compared with nontransgenic control animals. PTK6 activates signal transducer and activator of transcription 3 (STAT3), and active STAT3 was detected in PTK6-positive mammary gland epithelial cells. Endogenous mouse PTK6 was not detected in the normal mouse mammary gland, but it was induced in mouse mammary gland tumors of different origin, including spontaneous tumors that developed in control mice, and tumors that formed in PTK6, H-Ras, ERBB2 and PyMT transgenic models. MMTV-PTK6 and MMTV-ERBB2 transgenic mice were crossed to explore crosstalk between PTK6 and ERBB2 signaling in vivo. We found no significant increase in tumor incidence, size or metastasis in ERBB2/PTK6 double transgenic mice. Although we detected increased proliferation in ERBB2/PTK6 double transgenic tumors, an increase in apoptosis was also observed. MMTV-PTK6 clearly promotes mammary gland tumorigenesis in vivo, but its impact may be underrepresented in our transgenic models because of induction of endogenous PTK6 expression.
\end{abstract}

Oncogenesis (2013) 2, e81; doi:10.1038/oncsis.2013.43; published online 9 December 2013

Subject Categories: Molecular oncology

Keywords: PTK6; BRK; Sik; STAT3; ERBB2

\section{INTRODUCTION}

In spite of recent advances, breast cancer remains the second leading cause of death for women in the United States. ${ }^{1}$ The movement toward targeted therapies has seen the development of drugs to block the function of proteins associated with cancer progression and poor survival rates, including tyrosine kinases. Protein tyrosine kinase 6 (also called breast tumor kinase or BRK) is a tyrosine kinase that promotes growth factor signaling, and proliferation, migration and survival of breast cancer cells (for reviews $s^{2-6}$ ). It was identified in human metastatic breast cancer $^{7}$ and is overexpressed in the majority of human breast cancers and in most breast tumor cell lines. ${ }^{8-10}$ Its expression in high grade $E R(+)$ luminal $B$ tumors was associated with poor outcomes. ${ }^{11}$ The correlation between PTK6 and ERBB2 overexpression in invasive human ductal breast carcinomas ${ }^{9,12-14}$ and the finding that PTK6 may cooperate with ERBB2 to promote breast tumor cell growth ${ }^{14}$ raises the possibility that targeting PTK6 along with ERBB receptors might offer a therapeutic advantage. ${ }^{3,15}$

Functions of PTK6 in normal epithelia are distinct from its roles in cancer. PTK6 is expressed throughout the alimentary canal and in the skin in differentiated epithelial cells, ${ }^{16}$ and has been shown to promote differentiation of small intestinal enterocytes ${ }^{17}$ and keratinocytes. ${ }^{18,19}$ Interestingly, although PTK6 expression and functions in normal epithelia suggested it might have tumor suppressor roles, disruption of the mouse Ptk6 gene conferred resistance to carcinogens and impaired activation of the signal transducer and activator of transcription 3 (STAT3) transcription factor in the mouse colon. STAT3, a transcription factor that has essential roles in the development of a variety of tumor types, is a substrate of PTK6 and its activation is promoted by tyrosine phosphorylation. $^{20,21}$

To explore contributions of PTK6 to the development of breast cancer in vivo, we generated multiple lines of transgenic mice containing the human PTK6 gene expressed under control of the mouse mammary tumor virus (MMTV) long terminal repeat (LTR). We determined that the constitutive ectopic expression of PTK6 led to an $\sim 2.4$-fold increase in tumor development as animals aged, as well as enhanced STAT3 activation in transgenic mammary glands and tumors. Although expression of PTK6 has not been reported in normal mouse mammary gland, ${ }^{22}$ its expression was induced in mouse mammary gland tumors highlighting similarities between the human disease and mouse models. Induction of endogenous PTK6 may partially mask the activities of ectopic transgenic PTK6. We examined cell and proliferation and apoptosis within the mammary gland tumors that formed in transgenic and control mice. In addition, we examined potential synergy between PTK6 and ERBB2 signaling in mammary gland tumorigenesis and metastasis in vivo. 


\section{RESULTS}

Production and characterization of MMTV-PTK6 transgenic mice Induction of PTK6 expression in human breast tumors led us to hypothesize that ectopic expression of human PTK6 might promote mammary gland tumorigenesis in mice. To generate MMTV-PTK6 transgenic animals, PTK6 coding sequences were cloned into an expression vector containing the MMTV LTR promoter $^{23}$ (Figure 1a). The MMTV promoter has been extensively used to target transgene expression to the mammary gland in vivo. ${ }^{24}$ We determined that the MMTV-PTK6 construct, which is inducible by dexamethasone in tissue culture cell lines, could be expressed in mouse normal murine mammary gland (NMuMG) cells at levels comparable to that observed for PTK6 in human breast tumor cell lines (Figure 1b, NMuMG $+\mathrm{Tg}$ ).

Vector sequences were removed from the MMTV-PTK6 expression cassette before its microinjection into fertilized FVB/N eggs. Several transgenic founder mice were identified and three of these were used to develop lines for further analysis. The B28, B33 and B35 lines express low, medium and high levels of human PTK6 mRNA (Figure 1c) and protein (Figure 1d), respectively. The MMTV LTR drives transgene expression in the mammary gland of virgin adult, pregnant and postpartum mice, ${ }^{25-27}$ and expression occurs in the ductal and alveolar cells of the mammary gland. ${ }^{24}$ Using immunohistochemistry (Figure 1e), we detected transgene expression in mammary glands from both the virgin and multiparous female mice. Ectopic human PTK6 was detected in the nuclei and cytoplasm of mammary gland epithelial cells in all three established transgenic lines.

PTK6 promotes tumorigenesis in the mouse mammary gland Three independent mouse PTK6 transgenic lines were maintained and monitored for spontaneous tumorigenesis over a 2.5-year period. MMTV-PTK6 transgenic mice developed more than twice as many tumors as nontransgenic littermate controls, with an average latency of 21 months. However, tumors that formed in the PTK6 transgenic and nontransgenic control mice were similar in size and histology. Data are summarized in Table 1 and Figure 2.

Hyperplastic alveolar nodules were observed in PTK6 transgenic animals as early as 70 weeks of age, and were frequently detected in aging animals (Figures $3 a-c$ ). Transgenic mice developed multiple mammary gland tumors. An example of a mouse from the B33 line with tumors in its right inguinal and left thoracic mammary is shown at 105 weeks of age (Figure 3d). Ectopic PTK6 expression was detected in nulliparous and multiparous mammary glands and tumors using a human PTK6-specific antibody (Figures 3e-h).

Active ectopic PTK6 promotes STAT3 activation in normal mammary gland and mammary gland tumors

Activation of PTK6 can be monitored using an antibody specific for phosphorylation of tyrosine residue 342 (P-Y342) located in its catalytic domain. We expressed wild-type human PTK6, which may or may not be active, and has both kinase-dependent and independent functions. Using immunofluorescence, we examined PTK6 activation in mammary glands of transgenic mice $(\mathrm{Tg})$ and nontransgenic (NT) controls. Active PTK6 (P-Y342) can be detected by 12 weeks of age, with levels increasing and becoming more membrane associated at 40 weeks of age (Figure 4, top panels).

The STAT3 transcription factor is a substrate of PTK6. ${ }^{20}$ In addition to playing distinct roles in mammary gland development and involution, STAT3 promotes expression of genes that regulate cell proliferation, survival and tumor metastasis in the mammary gland (reviewed $\mathrm{in}^{28}$ ). Phosphorylation of STAT3 on tyrosine residue 705 (P-Y705) promotes its dimerization and activation, and a

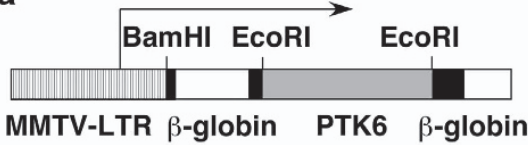

C

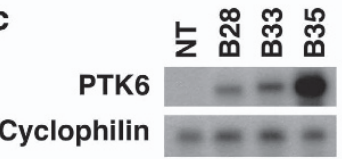

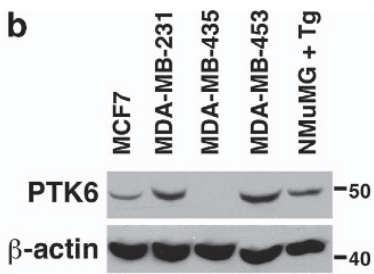

d

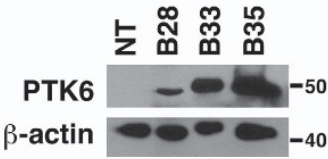

e
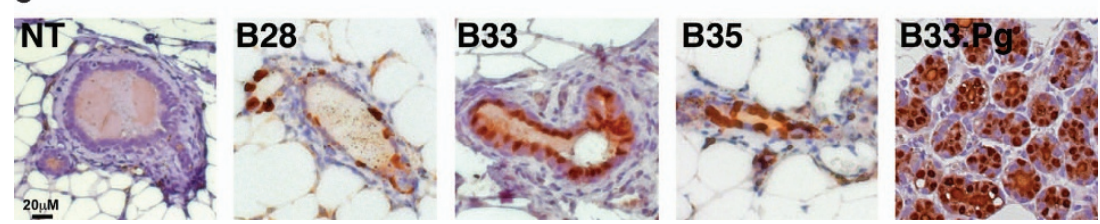

Figure 1. Generation of MMTV-PTK6 transgenic mice. (a) A schematic diagram of the MMTV-PTK6 construct is shown. A $2.2 \mathrm{~kb}$ human PTK6 complementary DNA (gray region) was inserted into the third exon of the rabbit $\beta$-globin gene under the control of the MMTV LTR (striped region). (b) Expression of the MMTV-PTK6 construct transfected into NMuMG cells stimulated with dexamethasone. Transgenic PTK6 protein levels (NMuMG + Tg) are comparable to that produced in human breast cancer cell lines MCF7, MDA-MB-231 and MDA-MB-453. PTK6 was not detected in the MDA-MB-435 cell line. Expression of $\beta$-actin was examined as a loading control. (c) Ribonuclease protection assays were performed with RNAs prepared from mammary glands of three transgenic lines (B28, B33 and B35) and nontransgenic control mice (NT). PTK6 mRNA was detectable in the three transgenic lines but not in NT animals. Mouse cyclophilin was used as loading control. (d) Ectopic PTK6 expression was detected in transgenic mammary glands by immunoblotting. Levels of ectopic PTK6 protein expression correlated with the levels of PTK6 mRNA shown in c. (e). Immunohistochemistry demonstrates expression of ectopic human PTK6 in the transgenic mammary gland epithelial cells, as shown in the virgin animals (B28, B33 and B35) and pregnant animals (B33.Pg). The nontransgenic mammary gland stained negative for PTK6. Size bar $=20 \mu \mathrm{m}$. 
Table 1. Tumor occurrence and latency in PTK6 transgenic and nontransgenic (NT) animals

\begin{tabular}{lccccc}
\hline $\begin{array}{l}\text { Transgenic } \\
\text { line }\end{array}$ & $\begin{array}{c}\text { Number } \\
\text { of } \\
\text { tumors }\end{array}$ & $\begin{array}{c}\text { Number } \\
\text { of } \\
\text { animals }\end{array}$ & $\begin{array}{c}\text { Tumor } \\
\text { occurrence } \\
\text { (\%) }\end{array}$ & $\begin{array}{c}\text { Average } \\
\text { tumor } \\
\text { weight } \\
(g)\end{array}$ & $\begin{array}{c}\text { Average } \\
\text { latency } \\
\text { (weeks) }\end{array}$ \\
\hline NT Controls & 5 & 104 & 4.81 & $3.7 \pm 1.1$ & 93 \\
PTK6 B28 & 5 & 49 & 10.2 & $4.9 \pm 2.6$ & 88 \\
PTK6 B33 & 7 & 65 & 10.77 & $2.6 \pm 2.0$ & 92 \\
PTK6 B35 & 3 & 22 & 13.64 & $3.6 \pm 1.2$ & 88 \\
\hline
\end{tabular}

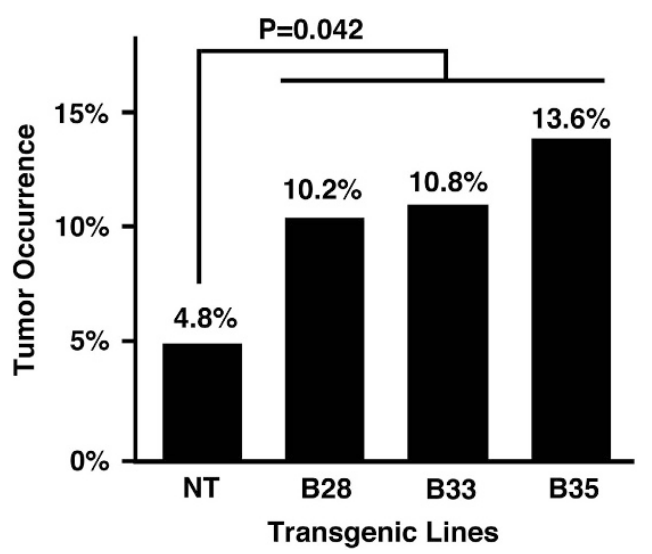

Figure 2. PTK6 transgenic animals display increased susceptibility to mammary gland tumor development. Nontransgenic FVB/N control and three independently developed MMTV-PTK6 transgenic mouse lines were maintained for 2.5 years and mammary gland tumorigenesis was monitored. Transgenic mice developed multiple mammary gland tumors during this period, and nontransgenic animals also developed spontaneous mammary gland tumors. The mammary gland tumor occurrence was shown as the percentage of animals that developed breast tumors relative to the total animal number. The Cochran-Armitage Trend test validated the hypothesis that increased numbers of MMTV-PTK6 transgenic animals develop mammary gland tumors than nontransgenic mice $(P=0.042)$.

was impaired in the normal mouse colon and human colon cancer cells following PTK6 knockout and knockdown, respectively. ${ }^{29}$ We found that levels of active P-Y705 STAT3 correlated with expression of the PTK6 transgene (Figure 4, bottom panels). Nuclear localization of active STAT3 was prominent in noninvoluting transgenic mammary glands at 40 weeks of age, but was not detected in nontransgenic controls.

Prominent activation of STAT3 was detected in tumors from PTK6 transgenic mice using both immunohistochemistry and immunoblotting (Figure 5). Localization of active PTK6 (P-Y342) at the plasma membrane correlated with increased activation and nuclear localization of STAT3 (P-Y705) (Figure 5a, Tg1), whereas the activation of PTK6 in the nucleus did not lead to significant activation and nuclear localization of STAT3 (Figure 5a, Tg2). Total cell lysates were prepared from tumors that formed in the three independently derived transgenic strains B28, B33, B35, and nontransgenic (NT) mice. Immunoblotting was performed with antibodies specific for active STAT3 (P-Y705), total STAT3, active PTK6 (P-Y342), total human PTK6 and $\beta$-actin as a control. The antibody used to detect PTK6 expressed from the transgene is specific for the human protein and does not recognize mouse PTK6. Each lane represents a tumor that formed in an individual mouse of the indicated strain. A significant increase in STAT3 activation (P-Y705) was detected in individual tumors from PTK6 transgenic mice compared with tumors that developed in nontransgenic controls (Figure 5b).

Induction of PTK6 in mouse tumors of different origins Immunoblotting and immunocytochemistry using anti-mouse PTK6 antibodies demonstrate PTK6 expression in tumors. We did not detect expression of endogenous PTK6 in the normal nontransgenic mouse mammary gland (Figures 6a-d; NT MG). However, endogenous mouse PTK6 expression is induced in a variety of mouse mammary gland tumors, including spontaneous tumors that form in nontransgenic mice (Figure 6a, NT TU), and tumors from transgenic mice that express human PTK6 (Figures 6a and e), ERBB2 (Figures $6 \mathrm{~b}$ and e), activated H-RAS (Figures $6 \mathrm{c}$ and e) or polyoma Middle T (Figures $6 \mathrm{~d}$ and e) in the mammary gland. The antibody used to detect mouse PTK6 was generated from a carboxy-terminal peptide that is not conserved between human and mouse, and the anti-human and anti-mouse PTK6 antibodies used are species specific. Interestingly, diverse patterns of PTK6 intracellular localization were observed, although many tumors displayed nuclear endogenous PTK6 localization. These data indicate that induction of PTK6 in breast cancer is conserved between humans and mice.

Enhanced proliferation is counteracted by increased apoptosis in ERBB2/PTK6 double transgenic mice

Several studies have indicated that PTK6 and ERBB2 are coexpressed in human breast tumors and PTK6 promotes ERBB2 oncogenic signaling in human breast tumor cell lines. ${ }^{14,15,30,31} \mathrm{We}$ hypothesized that introduction of MMTV-PTK6 would accelerate and/or augment ERBB2-induced mammary gland tumorigenesis in the mouse. We crossed the MMTV-PTK6 transgenic strains with the MMTV-ERBB2 line, which expresses the activated rat ErbB2 (c-neu) gene and is prone to developing mammary gland tumors. In our colony, $\sim 80 \%$ of MMTV-ERBB2 transgenic mice develop mammary gland tumors within 8 months. Coexpression of activated ERBB2 and PTK6 did not significantly influence the occurrence or size of tumors that developed. Unexpectedly, regression analysis suggested that PTK6 expression may delay tumor initiation and increase latency (Figure 7a).

To examine the impact that coexpressing PTK6 with ERBB2 has on proliferation, we examined 5-bromo-2'-deoxyuridine (BrdU) incorporation in tumors. Although tumor size was not increased (Figure 7c), a significant increase in cell proliferation was detected in tumors that formed in the double ERBB2/PTK6 transgenic mice, compared with single ERBB2 transgenic animals (Figure 7b). To determine whether an increase in programmed cell death might offset the increase in cell proliferation observed, terminal deoxynucleotidyl transferase dUTP nick end labeling assays were performed to detect apoptotic cells. Increased levels of terminal deoxynucleotidyl transferase dUTP nick end labeling-positive apoptotic cells were detected in ERBB2/PTK6 double transgenic mice (Figure 7d). Increased apoptosis could counteract the observed increase in cell proliferation and explain the lack of increased tumor size in vivo.

ERBB2-induced tumors metastasize to the lung in transgenic mice (reviewed $\mathrm{in}^{32}$ ). Lungs of ERBB2 and ERBB2/PTK6 animals were harvested from animals with tumors that had reached humane end point size, cut into $0.5 \times 0.5 \mathrm{~cm}^{2}$ pieces, fixed, embedded in paraffin and sectioned. Sections were stained with hematoxylin and eosin, and tumor emboli were counted and normalized with the area of the lung (represented by the number of $0.5 \times 0.5 \mathrm{~cm}^{2}$ lung pieces). Metastases were quantitated in 12 mice of each genotype (ERBB2 versus ERBB2/PTK6 transgenics), and correlations of lung metastasis with animal age and primary mammary gland tumor weight were analyzed (Figures 7e and f). 


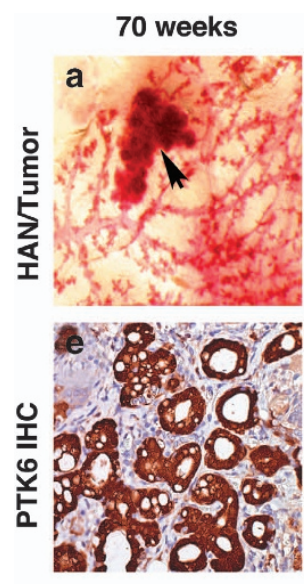

Nulliparous
95 weeks
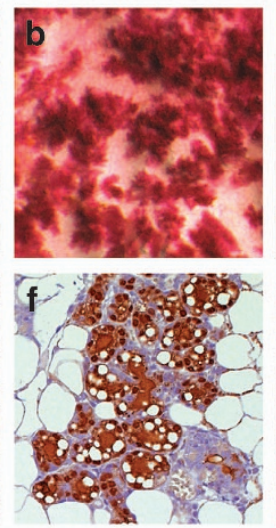

Nulliparous
100 weeks
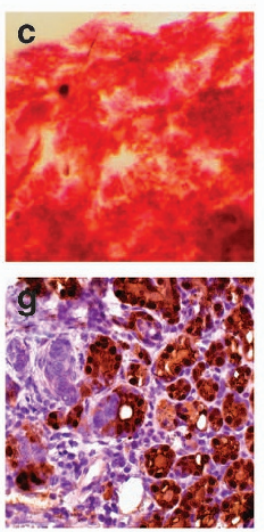

Multiparous
105 weeks
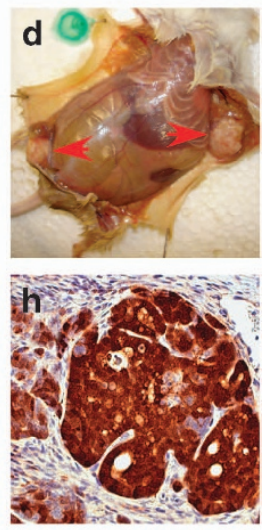

Multiparous

Figure 3. MMTV-PTK6 transgenic animals develop neoplastic hyperplasia and mammary gland tumors. Transgenic animals developed mammary gland lesions and mammary gland tumors at older age. Whole mount staining was performed on the thoracic mammary glands $(\mathbf{a}-\mathbf{c})$ of transgenic animals. Age and parous status were noted in the figure. Hyperplastic alveolar nodules (HAN) (black arrow) were observed in animals as early as 70 weeks of age, and were frequently detected in the aging animals (a-c). Multiple mammary gland tumors (red arrow) were found in the animal of 105 weeks (d). Immunohistochemistry performed on corresponding mammary glands confirmed PTK6 transgene expression (e-h).
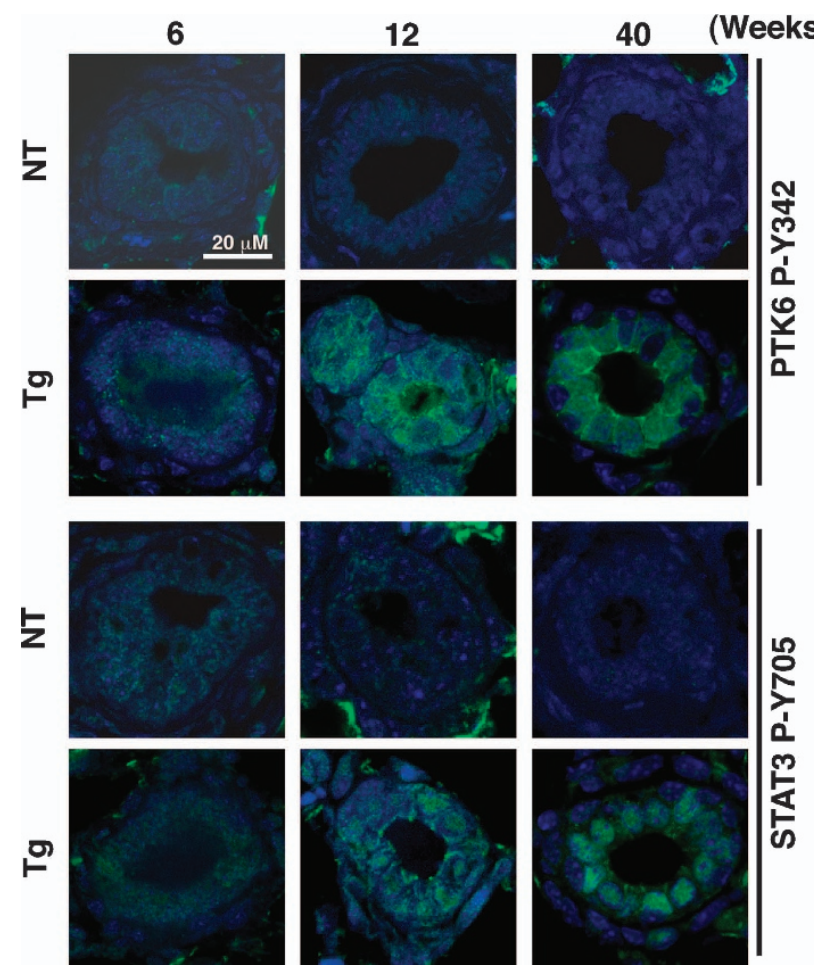

Figure 4. Ectopic PTK6 is active and promotes STAT3 phosphorylation at tyrosine residue 705 in the mouse mammary gland. Immunofluorescence assays were performed on mammary gland serial sections from age-matched nontransgenic (NT) or transgenic (Tg) animals using antibodies specific for active PTK6 (P-Y342) and active STAT3 (P-Y705). PTK6 was not expressed in prepubescent transgenic animals up to 6 weeks of age, and STAT3 phosphorylation was minimal. Upon maturity, PTK6 was expressed and activated in Tg mammary glands, and STAT3 phosphorylation and translocation to the nucleus was observed (12 weeks of age). At 40 weeks of age, ectopic PTK6 remained active and STAT3 displayed activating phosphorylation and nuclear localization in $\mathrm{Tg}$ animals, but this phosphorylation was not detected in NT mammary glands. Primary antibody binding was detected with fluorescein isothiocyanate (green) and sections were counterstained with DAPI (blue). The size bar represents $20 \mu \mathrm{m}$.
We did not detect a significant difference in the timing or size of metastases between the two groups.

\section{DISCUSSION}

Our data, obtained by characterizing multiple independent lines of MMTV-PTK6 transgenic mice, indicate that PTK6 promotes mammary gland tumorigenesis in vivo, but it is not a strong oncogenic driver. We detected an average 2.4-fold increase in tumor formation in virgin and multiparous animals compared with wild-type control FVB/N mice. However, in contrast to the MMTVERBB2 transgenic line used in these studies, tumor formation was modest. About $80 \%$ of the MMTV-ERBB2 mice developed tumors within 8 months compared with tumor formation in $10-13.6 \%$ of MMTV-PTK6 mice after 20 months. A previous study utilizing the whey acidic protein promoter to drive PTK6 expression in the mouse mammary gland reported a three fold higher incidence of tumor development in multiparous mice. ${ }^{33}$ Our study utilizing the MMTV promoter to drive PTK6 expression supports these findings.

Overexpression of PTK6 under control of the whey acidic protein promoter revealed delayed mammary gland involution that was associated with increased prosurvival signaling. ${ }^{33}$ We did not detect any obvious changes in mammary gland development or involution in our MMTV-PTK6 transgenic lines. The differences between the two models could be due to distinctions in the timing and pattern of PTK6 transgene expression as a consequence of using different promoters.

PTK6 is expressed in a high percentage of human breast tumors, and its activities in cancer have been most extensively examined in breast cancer cell lines. A variety of studies indicate that PTK6 stimulates signaling by multiple ERBB receptor family members. ${ }^{9,14,34-36}$ ERBB family kinases participate in the activation of signal transducers and activators of transcription (STATs) that regulate tumorigenesis, and direct roles for PTK6 in the activation of STAT3 ${ }^{20,37,38}$ and STAT5 $b^{39}$ have been reported. We show that STAT3 activation is increased in MMTV-PTK6 transgenic mammary glands and tumors (Figures 4 and 5) and could contribute to the increase in tumor formation observed in these mice. STAT3 contributes to development of a variety of cancers and was shown to regulate the growth of stem-like cells in human breast tumors. ${ }^{40}$ Inhibitors of STAT3 activity inhibited breast cancer cell growth. ${ }^{41}$ Interestingly, a tumor promoting the role for PTK6 was identified in colon cancer; Ptk6-null mice were resistant to an 
a
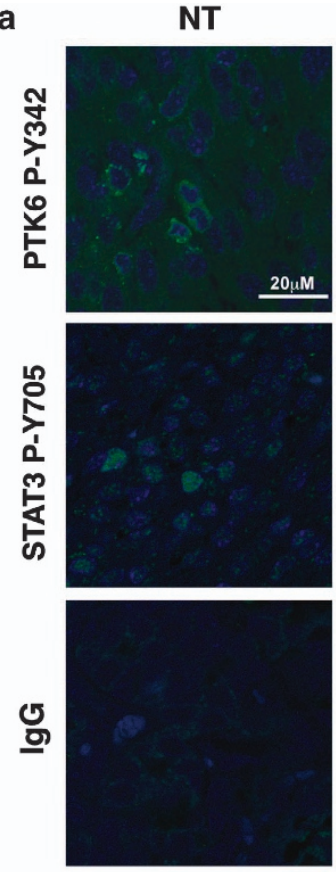

$\operatorname{Tg} 1$
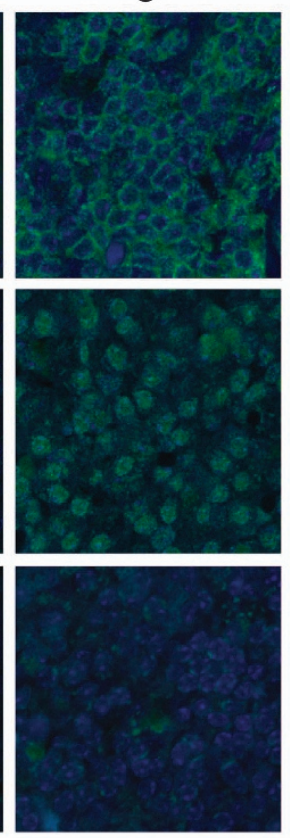
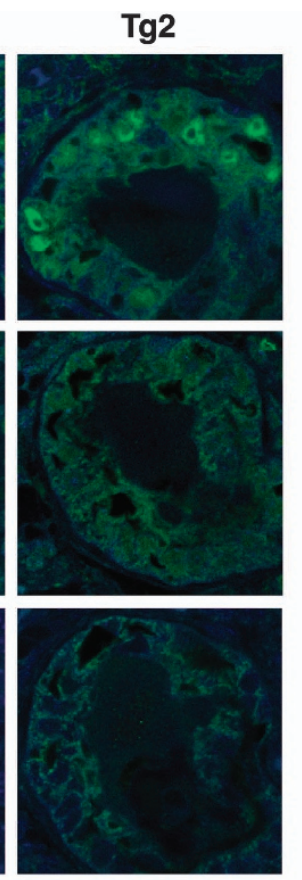

b
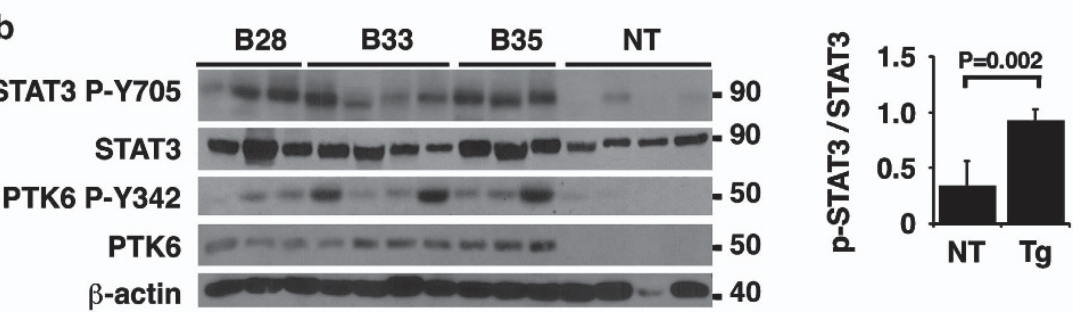

Figure 5. Active PTK6 and STAT3 are expressed in mouse mammary gland tumors. (a) Mammary gland tumors that developed in nontransgenic (NT) and PTK6 transgenic lines (Tg) were analyzed for the expression of active PTK6 and active STAT3 using immunofluorescence. Morphologically similar areas of NT and Tg tumors are shown. P-PTK6 and P-STAT3 signals were low and sporadic in NT tumors. Membrane-associated active PTK6 (P-Y342) correlated with active nuclear STAT3 (P-Y705) in tumors from PTK6 transgenic mice (Tg1). Active PTK6 could be found at the membrane (Tg1) or sometimes within the nucleus (Tg2). The tumor in Tg1 was an adenocarcinoma composed of small glandular structures with small lumens consistent with an acinar pattern, and the nuclear-activated PTK6 appeared in the acinar cells. Background staining was monitored using immunoglobulin G (lgG) as a control. The scale bar represents $20 \mu \mathrm{m}$. (b) Immunoblotting was performed with total cell lysates prepared from tumors isolated from multiple B28, B33 and B35 animals, as well as tumors that developed in nontransgenic control mice. Each lane represents a unique tumor sample from an individual mouse. STAT3 activation was consistently observed in tumor samples from PTK6 transgenic mice. For quantitation, P-STAT3 levels were normalized to total STAT3 levels in nontransgenic and transgenic mice (right panel). Immunoblotting for PTK6 was performed using an antibody specific for the human protein expressed by the transgene.

azoxymethane/dextran sodium sulfate tumorigenesis protocol and displayed reduced levels of activated phospho-STAT3. ${ }^{42}$ PTK6 was also shown to promote epidermal growth factorinduced STAT3 activation in human colon cancer cells. ${ }^{42}$

PTK6 is not expressed in the normal mouse mammary gland, ${ }^{22,43}$ but here we show that it is induced in mouse mammary gland tumors of different origins. PTK6 and ERBB2 are coexpressed in human tumors, and it has been suggested that PTK6 promotes cell proliferation and survival of ERBB2-positive tumors. Orthotopic transplantation of an immortalized pluripotent mouse mammary epithelial cell line engineered to overexpress activated ERBB2 alone or activated ERBB2 plus PTK6 revealed reduced latency for tumor development when both ERBB2 and PTK6 were overexpressed. ${ }^{14}$ In our studies, we detected increased proliferation in bitransgenic PTK6/ERRB2 mammary gland tumors, but also detected increased apoptosis that could counterbalance this increased proliferation. We previously determined that PTK6 promotes stress-induced apoptosis of nontransformed cells. ${ }^{42,44,45}$
PTK6 expression in normal tissues is developmentally regulated and coincides with epithelial cell differentiation. ${ }^{16-18}$ Disruption of the Ptk6 gene in the mouse revealed unique roles for this tyrosine kinase in promoting intestinal epithelial cell differentiation ${ }^{17}$ and stress-induced apoptosis. ${ }^{42,45}$ PTK6 may also have distinct functions in normal and transformed mammary epithelial cells. For example, although PTK6 promotes epidermal growth factorinduced proliferation in several breast cancer cell lines, it inhibited epidermal growth factor-induced proliferation in human telomerase reverse transcriptase immortalized human mammary gland epithelial cells. ${ }^{5}$ It is possible that poorly understood growth-inhibiting functions of PTK6 in normal mammary gland epithelial cells could have a role in delaying tumor initiation in the bitransgenic ERBB2/PTK6 mice.

Although our in vivo data do not demonstrate synergy between transgenic PTK6 and ERBB2, we cannot disregard contributions of endogenous PTK6. It is possible that induction of endogenous mouse PTK6 is sufficient to stimulate tumorigenesis and masks tumor promoting functions of ectopic transgenic human PTK6 


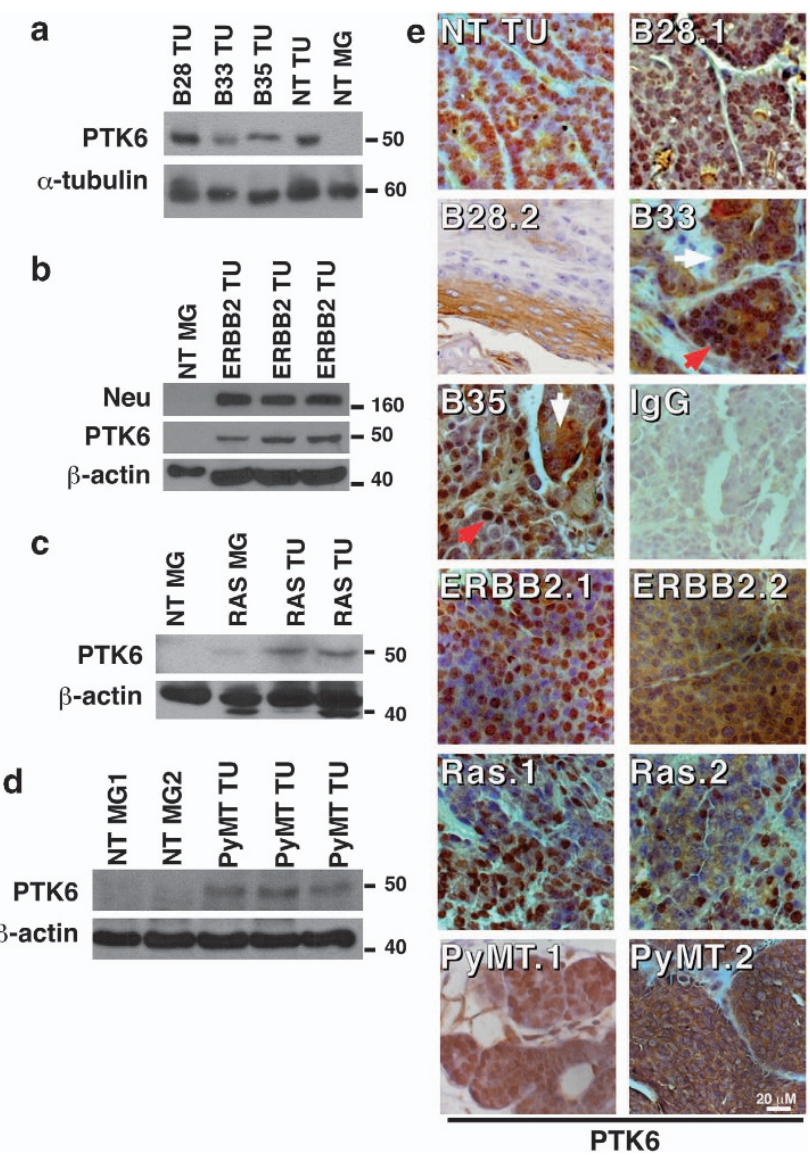

Figure 6. Endogenous mouse PTK6 is induced in mammary gland tumors of different origins. (a-d) Mouse PTK6 protein expression was detected using immunoblotting, and an antibody specific for mouse PTK6 that does not cross-react with the human PTK6 encoded by the transgene. Nontransgenic mammary gland was used as negative control. Expression of $\alpha$-tubulin or $\beta$-actin was examined as loading controls. Induction of the endogenous PTK6 protein was detected in all mouse mammary gland tumors examined, including tumors that formed in nontransgenic control mice (NT TU), MMTV-PTK6 (a), MMTV-ERBB2 (b), MMTV-Ha-Ras (c) MMTV-PyMT (d) transgenic animals. (e) Immunohistochemistry as used to examine endogenous PTK6 expression in mouse tumors of different origins. PTK6 is predominately nuclear in more differentiated acinar cells (NT, PyMT1) and in areas of tumors from different transgenic strains (B28.1, ERBB2.1 and Ras.1), but it can also be cytoplasmic as in ERBB2.2, Ras.2 and PyMT.2. Variation in PTK6 intracellular localization is sometimes observed in adjacent regions of the same tumors, as indicated by red (nuclear) and white (cytoplasmic/membrane) arrows in B33 and B35. Besides the neoplastic epithelial cells, endogenous PTK6 was also detected in the cytoplasm of cuboidal shaped epithelial cells and in metaplastic keratin producing squamous epithelial cells lining the ductules (B28.2). Sections were stained with normal rabbit IgG as a negative control (lgG).

in vivo. Disruption of the endogenous Ptk6 gene in ERBB2 transgenic mice would allow us to determine whether PTK6 has an essential role in ERBB2-induced tumorigenesis. Characterization of different mouse models of breast cancer lacking Ptk6 will be required to fully ascertain PTK6 contributions to mouse mammary gland tumorigenesis in vivo.

PTK6 is structurally related to SRC-family kinases, and has amino-terminal $\mathrm{SH} 2$ and $\mathrm{SH} 3$ protein-protein association domains and a carboxyl-terminal catalytic domain. However, unlike SRCfamily kinases, PTK6 lacks an SH4 domain and is not myristoylated/palmitoylated. ${ }^{2}$ PTK6 also lacks a nuclear localization signal.
Thus, it displays flexibility in its intracellular localization and has different functions in the nucleus and at the plasma membrane. ${ }^{6}$ In normal prostate cells, total and active PTK6 is concentrated in epithelial cell nuclei, but nuclear localization is lost in prostate tumors. ${ }^{46}$ Knockdown of cytoplasmic/membrane-associated PTK6 proved to be growth inhibiting, whereas reintroduction of PTK6 into the nucleus also inhibited growth prostate cancer PC3 cells. ${ }^{47}$ Targeting PTK6 to the cell membrane by addition of a myristoylation/palmitoylation signal resulted in oncogenic signaling. ${ }^{48,49}$ Ectopic expression of membrane-targeted PTK6 was sufficient to transform Src/Yes/Fyn - / - mouse embryonic fibroblasts. $^{50}$ Interestingly, active endogenous PTK6 was associated with the membrane Pten-null mouse prostates. ${ }^{50,51}$ Enhanced coexpression of membrane-associated growth factor receptors such as ERBB2 with PTK6 might bring PTK6 to the membrane in the absence of amino-terminal myristoylation/ palmitoylation, leading to its activation and induction of oncogenic signaling. However, in ERBB2 transgenic mammary glands, endogenous mouse PTK6 was often detected in the nucleus (Figure 6e), whereas most ERBB2 is membrane associated.

PTK6 substrates include a number of proteins involved in regulating the epithelial mesenchymal transition including $\mathrm{AKT}^{5},{ }^{52}$ p130CAS ${ }^{53}$ and FAK. ${ }^{50}$ We determined that membrane-targeted overexpression of PTK6 in prostate cells promotes the epithelial mesenchymal transition and tumor metastasis. ${ }^{51}$ PTK6 was also recently reported to have a role in the epithelial mesenchymal transition in breast cancer cells. ${ }^{31}$ Simultaneous knockdown of PTK6 and ERBB2 was reported to impair migration and proliferation of breast cancer cells in vitro. ${ }^{15}$ However, although we detected metastasis of ERBB2-positive tumors to the lungs of MMTV-ERBB2 mice, we did not find increased metastasis in ERBB2/ PTK6 double transgenic mice (Figure 7).

Our data indicate that PTK6 is induced in most mouse mammary gland tumors, regardless of the method used to induce the tumors. We detected induction of mouse PTK6 in spontaneous mouse mammary gland tumors as well as tumors caused by ectopic expression of ERBB2, activated RAS and PyMT (Figure 6). Recently, expression of endogenous PTK6 was also reported in mouse mammary gland tumors induced by the expression of an activated MET receptor transgene. ${ }^{54}$ Although PTK6 is overexpressed in most mouse and human breast cancer subtypes, its functions could differ depending on a variety of factors including its expression levels, intracellular localization, coexpression of other signaling molecules and cellular environment. Several studies suggest that targeting PTK6 may have therapeutic benefits in breast, ${ }^{11,15,31,55}$ colon $^{29}$ and prostate $^{6,51}$ cancer cells. However, earlier work also suggested a correlation between high PTK6 expression and differentiation (positive estrogen receptor status) ${ }^{56}$ as well as increased survival patient survival. ${ }^{13}$ More recent studies suggest that PTK6 has tumor suppressor functions in some cancers, including esophageal $^{57}$ and laryngeal ${ }^{58}$ tumors. Clearly, complexities of PTK6 signaling are not yet fully understood, and it will be necessary to determine whether targeting PTK6 expression has specific benefits for treatment in different molecularly defined subtypes of human breast cancer. Kinase inhibitors, along with targeted antibodies, represent some of the most effective anticancer therapies.

\section{MATERIALS AND METHODS}

Mice

To generate the MMTV-PTK6 construct, a $2.2 \mathrm{~kb}$ PTK6 complementary DNA fragment containing the coding region of human PTK6 was cloned into the EcoRI site of rabbit $\beta$-globin exon 3 of pKCR-MMTV LTR vector (a gift from Dr Robert J Coffey). ${ }^{23}$ MMTV-PTK6 transgenic mice were generated in the FVB/N inbred strain (Harlan Laboratories, Frederick, MD, USA) and FVB/N mice were used as controls. Tail DNA was subjected to PCR analysis with 
a

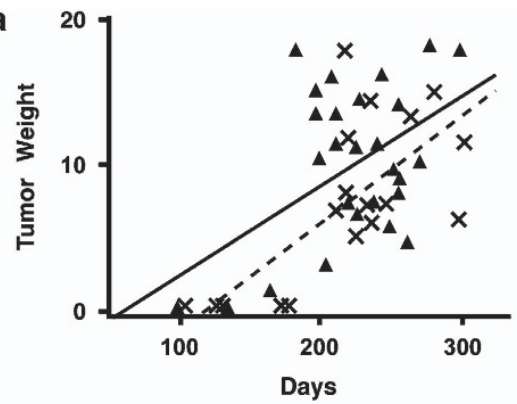

b

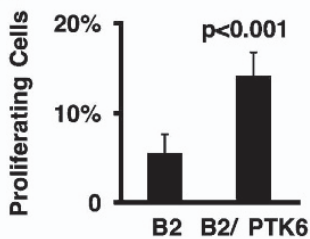

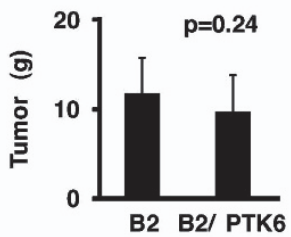

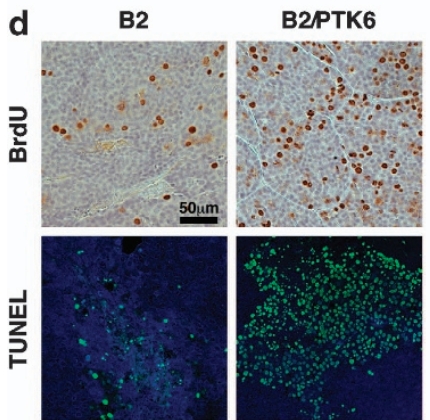

e

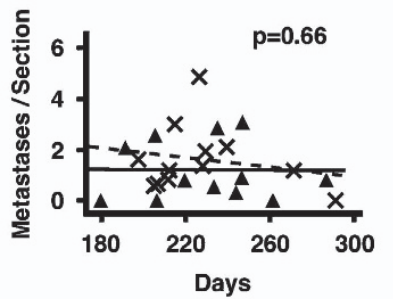

$\triangle \mathrm{B2}$ (Solid line)

X B2/ PTK6 (Dashed line) f

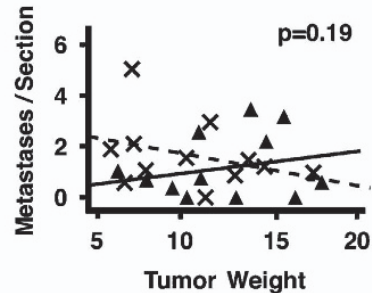

Figure 7. Transgenic expression of PTK6 in the MMTV-ERBB2 mouse model does not enhance ERBB2-driven tumorigenesis (a) Mammary gland tumors from ERBB2 $(B 2 ; n=28)$ and ERBB2/PTK6 mice $(B 2 / P T K 6 ; n=18)$ were harvested and analyzed at various time points; total tumor weight is plotted against age (days; see key below 7e and f). Tumor occurrence between ERBB2 and ERBB2/PTK6 animals did not show a statistically significant difference $(P=0.70)$. However, regression analysis suggested a delay in tumor initiation in ERBB2/PTK6 animals $(P<0.001)$. (b) Proliferation in tumors that developed in ERBB2 (B2; $n=10)$ and ERBB2/PTK6 (B2/PTK6; $n=10)$ transgenic mice was examined using BrdU labeling. ERBB2/PTK6 tumors exhibited higher levels of proliferation than ERBB2 tumors, even in tumors of the same size. The number of BrdU-labeled epithelial cells in ERBB2/PTK6 tumors is three fold higher than in ERBB2 tumors $(P<0.001)$, but the tumor size between these two groups is not significantly different $(P=0.24)(\mathbf{c})$. (d) Although ERBB2/PTK6 tumors display higher levels of proliferation (BrdU incorporation), they also exhibit increased apoptosis. Increased apoptosis is detected in tumors that formed in ERBB2/PTK6 double transgenic animals compared with tumors that formed in ERBB2 transgenic mice. Apoptosis was analyzed using the terminal deoxynucleotidyl transferase dUTP nick end labeling (TUNEL) assay in several pairs of equivalently sized ERBB2 and ERBB2/PTK6 tumors. (e, f) We examine lung metastases in single ERBB2 and double ERBB2/PTK6 transgenic mice and correlated the development of metastases with age (e) and primary mammary gland tumor weight (f) are shown. Linear regression models were fitted to the data and Wald tests were conducted to compare regression slopes between the ERBB2 (B2) and ERBB2/PTK6 (B2/PTK6) transgenic groups, resulting in P-values of 0.66 and 0.19 for (e) and (f), respectively, suggesting that the slopes from two experimental groups are not statistically different from each other.

primers that were specific for the transgene (forward: $5^{\prime}$ GCTATGTGCCCCACAACTACC-3', reverse: 5'-CCTGCAGAGCGTGAACTC-3'). Nulliparous females were never housed with males after weaning. Multiparous females were kept in breeding and underwent at least two, but generally three to four pregnancies. Mice showing signs of discomfort, weight loss or tumors larger than $2 \mathrm{~cm}$ in diameter were killed as they met end point criteria of the protocol approved by the UIC (University Isotope Committee) Institutional Animal Care and Use Committee.

MMTV-Ha-RAS (FVB.Cg-Tg(MMTV-vHaras)SH1Led/J, stock number 004363) and MMTV-ERBB2 mice (FVB-(MMTV-Erbb2)NK1Mul/J, stock number 005038), expressing activated ERBB2 were purchased from Jackson Laboratories (Bar Harbor, ME, USA). MMTV-PyMT-induced mouse mammary gland tumors were provided by Dr P Raychaudhuri (University of Illinois at Chicago).

\section{Cell culture and transfection}

The human breast cancer cell lines MCF7 (HTB-22), MDA-MB-231 (HTB-26) and MDA-MB-453 (HTB-131), human melanoma cell line MDA-MB-435S (HTB-129) and the mouse mammary epithelial cell line NMuMG (CRL-1636) were obtained from ATCC (American Type Culture Collection) and cultured according to the ATCC guidelines. Transfections of NMuMG cells were performed using Lipofectamine (Invitrogen Corp, Carlsbad, CA, USA). To induce MMTV-PTK6 expression, $0.1 \mu \mathrm{m}$ of dexamethasone (D8893, SigmaAldrich, St Louis, MO, USA) was added to the media $24 \mathrm{~h}$ before harvesting.

\section{Tissue preparation and analyses}

For whole mounts, mammary glands were harvested, spread on glass slides, air dried for $5 \mathrm{~min}$ and then fixed in Pen-fix solution (Richard-Allan Scientific, Kalamazoo, Ml, USA) for $24 \mathrm{~h}$. Samples were then passed through graded ethanols, acetone and rehydrated. Tissues were stained in carmine alum for 3 days and then dehydrated in ethanol followed by clearing in xylene (Fisher Scientific, Fair Lawn, NJ, USA). Stained whole mounts were stored in xylene during examination and were photographed using a dissection microscope.

For microscopic sections, tissues were fixed in $10 \%$ buffered formalin (Fisher Scientific) for $24 \mathrm{~h}$ and then transferred to $70 \%$ ethanol before routine processing. Paraffin-embedded tissues were sectioned at $5 \mu$ and stained with hematoxylin and eosin. Whole mount analysis, postmortem examination and histopathologic analysis of tissue sections were performed by a veterinary pathologist (S Ball-Kell, DVM and PhD).

RNA extraction and ribonuclease protection assays

Total RNA was isolated from animal tissues using TRIZOL reagent (GIBCO Invitrogen, CA, USA). Ribonuclease protection assays were performed as described previously ${ }^{43}$ using $\left[{ }^{32} \mathrm{P}\right] \alpha$-CTP-labeled antisense RNA probes. Mouse cyclophilin mRNA was used as loading control and RNA integrity indicator, and the mouse cyclophilin antisense probe was synthesized from pTRI-cyclophilin-mouse antisense control template (Ambion, Grand Island, NY, USA).

\section{Protein lysates and immunoblotting}

Fresh tissues were rinsed in phosphate-buffered saline and homogenized by tissue homogenizer (Polytron, PT-10, Kinematica, Lucerne, Switzerland) in Triton X-100 buffer (20 mм Hepes, pH 7.4, 1\% Triton X-100, $150 \mathrm{~mm} \mathrm{NaCl,}$ $1 \mathrm{~m}$ EDTA, pH 8.0, $1 \mathrm{~mm}$ EGTA, pH 8.0, $10 \mathrm{~mm}$ Na-pyrophosphate, $100 \mathrm{~mm}$ $\mathrm{NaF}, 5 \mathrm{~mm}$ iodoacetic acid, $1 \mathrm{~mm}$ sodium vanadate, $0.2 \mathrm{~mm}$ PMSF and proteinase inhibitor cocktail tablet (Roche Diagnostic, Indianapolis, IN, USA). 
Immunoblotting was performed as previously described. ${ }^{17}$ Polyvinylidene difluoride membranes were blocked in Tris-Buffered Saline Tween-20 solution $(150 \mathrm{~mm} \mathrm{NaCl}, 20 \mathrm{~mm} \mathrm{pH} 7.5$ Tris and $1 \%$ Tween-20) with $5 \%$ non-fat milk or bovine serum albumin (Sigma-Aldrich) for $1 \mathrm{~h}$ at room temperature, then incubated in primary antibody for $1 \mathrm{~h}$ at room temperature or overnight at $4{ }^{\circ} \mathrm{C}$ according to the antibody manufacturers' recommendations.

\section{Antibodies}

Anti-human PTK6 (C-18) and anti-mouse PTK6 (C-17) antibodies were purchased from Santa Cruz Biotechnology (Santa Cruz, CA, USA). Antiphospho-PTK6 Tyr-342 (P-Y342) antibody was purchased from Millipore (Bedford, MA, USA). Total STAT3 and phospho-STAT3 P-Y705 antibodies were purchased from Cell Signaling Technology (Danvers, MA, USA). Anti$\beta$-actin (AC-15) and anti- $\alpha$-tubulin were purchased from Sigma-Aldrich Sheep anti-mouse and donkey anti-rabbit antibodies were purchased from GE Healthcare Biosciences (Pittsburgh, PA, USA).

\section{Immunohistochemistry and immunofluorescence}

Immunohistochemistry was performed using the Vectastain $A B C$ kit (Vector Laboratories, Burlingame, CA, USA) and 3,3'-diaminobenzidine tetrahydrochloride tablets (Sigma-Aldrich). Samples were submerged in subboiling $0.01 \mathrm{~m}$ sodium citrate for $20 \mathrm{~min}$ for antigen retrieval. After the 3,3'diaminobenzidine tetrahydrochloride reaction, slides were stained with haematoxylin before proceeding to dehydration and mounting.

For immunofluorescence, slides were blocked with bovine serum albumin for an hour and incubated with antibodies at $4{ }^{\circ} \mathrm{C}$ overnight. After washing in TNT buffer $(0.1 \mathrm{~m}$ Tris- $\mathrm{HCl}, \mathrm{pH} 7.5,150 \mathrm{~mm} \mathrm{NaCl}, 0.05 \%$ Tween-20), slides were incubated with biotinylated anti-rabbit or antimouse secondary antibodies and then fluorescein isothiocyanate-conjugated avidin (Vector Laboratories).

\section{Proliferation and apoptosis assays}

Animals were injected intraperitoneally with BrdU (Sigma) in phosphatebuffered saline at $50 \mu \mathrm{g} / \mathrm{g}$ of body weight $2 \mathrm{~h}$ before killing. BrdU incorporation was detected using anti-BrdU (BD, San Jose, CA, USA) and the Mouse-on-Mouse (M.O.M) immunodetection Kit (Vector Laboratories). For each sample, five pictures were taken from different areas chose randomly to represent the average distribution of BrdU-positive cells.

Terminal deoxynucleotidyl transferase dUTP nick end labeling was performed with ApopTag Fluorescein In Situ Apoptosis Detection Kit (Millipore), all procedures were performed according to the manufacturer's protocol.

\section{Statistics}

Statistical analysis was performed in consultation with $\mathrm{HX}$, a statistician in the UIC Design and Analysis Core. Univariate analysis was initially conducted to summarize the tumor data. Categorical data are presented as percentages and Pearson $\chi^{2}$ tests are used to compare frequency distributions among different experimental groups. When appropriate, the more powerful Cochran-Armitage Trend tests are applied. Continuous data are presented as means and s.d.'s. Two-sample $t$-tests are used to compare the mean values between two groups. Linear regression models are fitted to the data to describe the relationship between normalized tumor size and age, and Wald tests are used to compare regression lines from different experimental groups. All analyses were performed using SAS statistical software version 9.2 (SAS Institute Inc., Cary, NC, USA) Densitometry analysis of immunoblotting results was performed with ImageJ 1.45s (NIH, Bethesda, MD, USA) ${ }^{59}$ Quantitative data are shown as the mean $\pm \mathrm{s}$.d. $P$-values were determined using the two-tailed Student's $t$ test (Microsoft Excel, 2010). A difference was considered statistically significant if the $P$-value was equal to or less than 0.05 .

\section{CONFLICT OF INTEREST}

The authors declare no conflict of interest.

\section{ACKNOWLEDGEMENTS}

L Tyner is supported by NIH grant RO1 DK044525 and an award from the Elsa U Pardee Foundation. $\mathrm{H}$ Xie receives support from the University of Illinois at Chicago Cancer Center and the Center for Clinical and Translational Science, funded in part by the NIH National Center for Research Resources, grant UL1TR000050. We thank Ms Priya Mathur and Dr Yu Zheng for providing helpful comments and editing the manuscript.

\section{AUTHOR CONTRIBUTIONS}

MP: Acquisition of data, analysis and interpretation of data, drafting the manuscript. SB-K: Acquisition of data and analysis and interpretation of data (Vet Pathologist) RRF: Design, acquisition of data. HX: Analysis and interpretation of data (Biostatistician). ALT: Design, analysis and interpretation of data, drafting the manuscript.

\section{REFERENCES}

1 ACS. Cancer Facts \& Figures 2013. American Cancer Society: Atlanta, GA, USA, 2013.

2 Serfas MS, Tyner AL. Brk Srm, Frk, and Src42A form a distinct family of intracellular Src-like tyrosine kinases. Oncol Res 2003; 13: 409-419.

3 Harvey AJ, Crompton MR. The Brk protein tyrosine kinase as a therapeutic target in cancer: opportunities and challenges. Anticancer Drugs 2004; 15 107-111.

4 Brauer PM, Tyner AL. Building a better understanding of the intracellular tyrosine kinase PTK6 - BRK by BRK. Biochim et biophysic acta 2010; 1806: 66-73.

5 Ostrander JH, Daniel AR, Lange CA. Brk/PTK6 signaling in normal and cancer cell models. Curr Opin Pharmacol 2010; 10: 662-669.

6 Zheng Y, Tyner AL. Context-specific protein tyrosine kinase 6 (PTK6) signalling in prostate cancer. Eur J Clin Invest 2013; 43: 397-404.

7 Mitchell PJ, Barker KT, Martindale JE, Kamalati T, Lowe PN, Page MJ et al. Cloning and characterisation of CDNAs encoding a novel non-receptor tyrosine kinase, brk, expressed in human breast tumours. Oncogene 1994; 9: 2383-2390.

8 Harvey AJ, Pennington CJ, Porter S, Burmi RS, Edwards DR, Court W et al. Brk protects breast cancer cells from autophagic cell death induced by loss of anchorage. Am J Pathol 2009; 175: 1226-1234.

9 Ostrander JH, Daniel AR, Lofgren K, Kleer CG, Lange CA. Breast tumor kinase (protein tyrosine kinase 6) regulates heregulin-induced activation of ERK5 and p38 MAP kinases in breast cancer cells. Cancer Res 2007; 67: 4199-4209.

10 Barker KT, Jackson LE, Crompton MR. BRK tyrosine kinase expression in a high proportion of human breast carcinomas. Oncogene 1997; 15: 799-805.

11 Irie HY, Shrestha Y, Selfors LM, Frye F, lida N, Wang Z et al. PTK6 regulates IGF-1induced anchorage-independent survival. PLoS One 2010; 5: e11729.

12 Born M, Quintanilla-Fend L, Braselmann H, Reich U, Richter M, Hutzler P et al. Simultaneous over-expression of the Her2/neu and PTK6 tyrosine kinases in archival invasive ductal breast carcinomas. J Pathol 2005; 205: 592-596.

13 Aubele M, Auer G, Walch AK, Munro A, Atkinson MJ, Braselmann H et al. (protein tyrosine kinase) -6 and HER2 and 4, but not HER1 and 3 predict long-term survival in breast carcinomas. Br J Cancer 2007; 96: 801-807.

14 Xiang B, Chatti K, Qiu H, Lakshmi B, Krasnitz A, Hicks J et al. Brk is coamplified with ErbB2 to promote proliferation in breast cancer. Proc Natl Acad Sci USA 2008; 105: 12463-12468.

15 Ludyga N, Anastasov N, Rosemann M, Seiler J, Lohmann N, Braselmann H et al. Effects of simultaneous knockdown of HER2 and PTK6 on malignancy and tumor progression in human breast cancer cells. Mol Cancer Res 2013; 11: 381-392.

16 Vasioukhin V, Serfas MS, Siyanova EY, Polonskaia M, Costigan VJ, Liu B et al. A novel intracellular epithelial cell tyrosine kinase is expressed in the skin and gastrointestinal tract. Oncogene 1995; 10: 349-357.

17 Haegebarth A, Bie W, Yang R, Crawford SE, Vasioukhin V, Fuchs E et al. Protein tyrosine kinase 6 negatively regulates growth and promotes enterocyte differentiation in the small intestine. Mol Cell Biol 2006; 26: 4949-4957.

18 Vasioukhin V, Tyner AL. A role for the epithelial-cell-specific tyrosine kinase Sik during keratinocyte differentiation. Proc Natl Acad Sci USA 1997; 94: 14477-14482.

19 Wang TC, Jee SH, Tsai TF, Huang YL, Tsai WL, Chen RH. Role of breast tumour kinase in the in vitro differentiation of HaCaT cells. $\mathrm{Br} J$ Dermatol 2005; 153: 282-289.

20 Liu L, Gao Y, Qiu H, Miller WT, Poli V, Reich NC. Identification of STAT3 as a specific substrate of breast tumor kinase. Oncogene 2006; 25: 4904-4912.

21 Gao Y, Cimica V, Reich NC. Suppressor of cytokine signaling 3 inhibits breast tumor kinase activation of STAT3. J J Biol Chem 2012; 287: 20904-20912.

22 Llor X, Serfas MS, Bie W, Vasioukhin V, Polonskaia M, Derry J et al. BRK/Sik expression in the gastrointestinal tract and in colon tumors. Clin Cancer Res 1999; 5: 1767-1777.

23 Matsui Y, Halter SA, Holt JT, Hogan BL, Coffey RJ. Development of mammary hyperplasia and neoplasia in MMTV-TGF alpha transgenic mice. Cell 1990; 61: 1147-1155.

24 Hennighausen L. Mouse models for breast cancer. Breast Cancer Res 2000; 2: 2-7. 
25 Sinn E, Muller W, Pattengale P, Tepler R, Wallace R, Leder P. Coexpression of MMTV/ v-Ha-ras and MMTV-/c-myc genes in transgenic mice. Cell 1987; 48: 465-475.

26 Bouchard L, Lamarre PJ, Tremblay PJ, Jolicoeur P. Stochastic appearance of mammary tumors in transgenic mice carrying the MMTV-/c-neu oncogene. Cell 1989; 57: 931-936.

27 Pierce DF, Johnson MD, Matsui Y, Robinson SD, Gold LI, Purchio AF et al. Inhibition of mammary duct development but not alveolar outgrowth during pregnancy in transgenic mice expressing active TGF-b. Genes Dev 1993; 7: 2308-2317.

28 Haricharan S, Li Y. STAT signaling in mammary gland differentiation, cell survival and tumorigenesis. Mol Cell Endocrinol (e-pub ahead of print 28 March 2013; doi:10.1016/jmce.2013.03.014).

29 Gierut JJ, Mathur PS, Bie W, Han J, Tyner AL. Targeting protein tyrosine kinase 6 enhances apoptosis of colon cancer cells following DNA damage. Mol Cancer Therap 2012; 11: 2311-2320.

30 Ludyga N, Anastasov N, Gonzalez-Vasconcellos I, Ram M, Hofler H, Aubele M. Impact of protein tyrosine kinase 6 (PTK6) on human epidermal growth factor receptor (HER) signalling in breast cancer. Mol Biosyst 2011; 7: 1603-1612.

31 Ai M, Liang K, Lu Y, Qiu S, Fan Z. Brk/PTK6 cooperates with HER2 and Src in regulating breast cancer cell survival and epithelial-to-mesenchymal transition. Cancer Biol Therapy 2013; 14: 237-245.

32 Ursini-Siegel J, Schade B, Cardiff RD, Muller WJ. Insights from transgenic mouse models of ERBB2-induced breast cancer. Nat Rev Cancer 2007; 7: 389-397.

33 Lofgren KA, Ostrander JH, Housa D, Hubbard GK, Locatelli A, Bliss RL et al. Mammary gland specific expression of Brk/PTK6 promotes delayed involution and tumor formation associated with activation of p38 MAPK. Breast Cancer Res 2011; 13: R89.

34 Kamalati T, Jolin HE, Mitchell PJ, Barker KT, Jackson LE, Dean CJ et al. Brk a breast tumor-derived non-receptor protein-tyrosine kinase, sensitizes mammary epithelial cells to epidermal growth factor. J Biol Chem 1996; 271: 30956-30963.

35 Kamalati T, Jolin HE, Fry MJ, Crompton MR. Expression of the BRK tyrosine kinase in mammary epithelial cells enhances the coupling of EGF signalling to PI 3-kinase and Akt, via erbB3 phosphorylation. Oncogene 2000; 19: 5471-5476.

36 Chen HY, Shen $\mathrm{CH}$, Tsai YT, Lin FC, Huang YP, Chen RH. Brk activates rac1 and promotes cell migration and invasion by phosphorylating paxillin. $\mathrm{Mol} \mathrm{Cell} \mathrm{BiO}$ 2004; 24: 10558-10572.

37 Ikeda O, Miyasaka Y, Sekine Y, Mizushima A, Muromoto R, Nanbo A et al. STAP-2 is phosphorylated at tyrosine- 250 by Brk and modulates Brk-mediated STAT3 activation. Biochem Biophys Res Commun 2009; 384: 71-75.

38 Ikeda O, Sekine Y, Mizushima A, Nakasuji M, Miyasaka Y, Yamamoto $C$ et al. Interactions of STAP-2 with Brk and STAT3 participate in cell growth of human breast cancer cells. J Biol Chem 2010; 285: 38093-38103.

39 Weaver AM, Silva CM. Signal transducer and activator of transcription 5b: a new target of breast tumor kinase/protein tyrosine kinase 6. Breast Cancer Res 2007; 9: R79.

40 Marotta LL, Almendro V, Marusyk A, Shipitsin M, Schemme J, Walker SR et al. The JAK2/STAT3 signaling pathway is required for growth of CD44(+)CD24(-) stem cell-like breast cancer cells in human tumors. J Clin Invest 2011; 121: 2723-2735.

41 Lin L, Hutzen B, Zuo M, Ball S, Deangelis S, Foust E et al. Novel STAT3 phosphorylation inhibitors exhibit potent growth-suppressive activity in pancreatic and breast cancer cells. Cancer Res 2010; 70: 2445-2454.

42 Gierut J, Zheng Y, Bie W, Carroll RE, Ball-Kell S, Haegebarth A et al. Disruption of the mouse protein tyrosine kinase 6 gene prevents STAT3 activation and confers resistance to azoxymethane. Gastroenterology 2011; 141: 1371-80 e2.

43 Haegebarth A, Heap D, Bie W, Derry JJ, Richard S, Tyner AL. The nuclear tyrosine kinase BRK/Sik phosphorylates and inhibits the RNA-binding activities of the Sam68-like mammalian proteins SLM-1 and SLM-2. J Biol Chem 2004; 279: 54398-54404.

44 Haegebarth A, Nunez R, Tyner AL. The intracellular tyrosine kinase Brk sensitizes non-transformed cells to inducers of apoptosis. Cell Cycle 2005; 4: 1239-1246.

45 Haegebarth A, Perekatt AO, Bie W, Gierut JJ, Tyner AL. Induction of protein tyrosine kinase 6 in mouse intestinal crypt epithelial cells promotes DNA damage-induced apoptosis. Gastroenterology 2009; 137: 945-954.

46 Derry JJ, Prins GS, Ray V, Tyner AL. Altered localization and activity of the intracellular tyrosine kinase BRK/Sik in prostate tumor cells. Oncogene 2003; 22: 4212-4220.

47 Brauer PM, Zheng Y, Wang L, Tyner AL. Cytoplasmic retention of protein tyrosine kinase 6 promotes growth of prostate tumor cells. Cell Cycle 2010; 9: 4190-4199.

48 le Kim H, Lee ST. Oncogenic functions of PTK6 are enhanced by its targeting to plasma membrane but abolished by its targeting to nucleus. J Biochem 2009; 146: 133-139.

49 Palka-Hamblin HL, Gierut JJ, Bie W, Brauer PM, Zheng Y, Asara JM et al. Identification of beta-catenin as a target of the intracellular tyrosine kinase PTK6. J Cell Sci 2010; 123(Pt 2): 236-245.

50 Zheng Y, Gierut J, Wang Z, Miao J, Asara JM, Tyner AL. Protein tyrosine kinase 6 protects cells from anoikis by directly phosphorylating focal adhesion kinase and activating AKT. Oncogene 2012; 32: 4304-4312.

51 Zheng Y, Wang Z, Bie W, Brauer PM, Perez-White BE, Li J et al. PTK6 activation at the membrane regulates epithelial-mesenchymal transition in prostate cancer. Cancer Res 2013; 73: 5426-5437.

52 Zheng Y, Peng M, Wang Z, Asara JM, Tyner AL. Protein tyrosine kinase 6 directly phosphorylates AKT and promotes AKT activation in response to epidermal growth factor. Mol Cell Biol 2010; 30: 4280-4292.

53 Zheng Y, Asara JM, Tyner AL. Protein-tyrosine Kinase 6 promotes peripheral adhesion complex formation and cell migration by phosphorylating p130 CRKassociated substrate. J Biol Chem 2012; 287: 148-158.

54 Regan Anderson TM, Peacock DL, Daniel AR, Hubbard GK, Lofgren KA, Girard BJ et al. Breast tumor kinase (Brk/PTK6) Is a mediator of hypoxia-associated breast cancer progression. Cancer Res 2013; 73: 5810-5820.

55 Harvey AJ, Crompton MR. Use of RNA interference to validate Brk as a novel therapeutic target in breast cancer: Brk promotes breast carcinoma cell proliferation. Oncogene 2003; 22: 5006-5010.

56 Zhao $\mathrm{C}$, Yasui $\mathrm{K}$, Lee $\mathrm{CJ}$, Kurioka $\mathrm{H}$, Hosokawa $\mathrm{Y}$, Oka $\mathrm{T}$ et al. Elevated expression levels of NCOA3, TOP1, and TFAP2C in breast tumors as predictors of poor prognosis. Cancer 2003; 98: 18-23.

57 Ma S, Bao JY, Kwan PS, Chan YP, Tong CM, Fu L et al. Identification of PTK6, via RNA sequencing analysis, as a suppressor of esophageal squamous cell carcinoma. Gastroenterology 2012; 143: 675-86 e1-12.

58 Liu XK, Zhang XR, Zhong Q, Li MZ, Liu ZM, Lin ZR et al. Low expression of PTK6/Brk predicts poor prognosis in patients with laryngeal squamous cell carcinoma. J Transl Med 2013; 11: 59.

59 Rasband WS. ImageJ. U S National Institutes of Health: Bethesda, MA, USA, 1997-2011; http://imagej.nih.gov/ij/.

(i) $\odot$ Oncogenesis is an open-access journal published by Nature Publishing Group. This work is licensed under a Creative Commons AttributionNonCommercial-NoDerivs 3.0 Unported License. To view a copy of this license, visit http://creativecommons.org/licenses/by-nc-nd/3.0/ 